\title{
Pointwise Estimates for Solutions to a System of Radiating Gas
}

\author{
Shikuan Mao, Yongqin Liu \\ School of Mathematics and Physics, North China Electric Power University, Beijing, China \\ Email: shikuanmao@ncepu.edu.cn,yqliu2@ncepu.edu.cn
}

Received August 22, 2013; revised September 8, 2013; accepted September 13, 2013

Copyright (C) 2013 Shikuan Mao, Yongqin Liu. This is an open access article distributed under the Creative Commons Attribution License, which permits unrestricted use, distribution, and reproduction in any medium, provided the original work is properly cited.

\begin{abstract}
In this paper we focus on the initial value problem of a hyperbolic-elliptic coupled system in multi-dimensional space of a radiating gas. By using the method of Green function combined with Fourier analysis, we obtain the pointwise decay estimates of solutions to the problem.
\end{abstract}

Keywords: Radiating Gas; Initial Value Problem; Pointwise Estimates

\section{Introduction}

In this paper we consider the initial value problem

$$
\begin{cases}u_{t}+a \cdot \nabla u^{2}+\operatorname{div} q=0, & x \in \mathbb{R}^{n}, t>0, \\ -\nabla \operatorname{div} q+q+\nabla u=0, & x \in \mathbb{R}^{n}, t>0, \\ u(x, 0)=u_{0}(x), & x \in \mathbb{R}^{n},\end{cases}
$$

here $a \in \mathbb{R}^{n}$ is a constant vector, $u=u(x, t)$ and $q=\left(q_{1}, \cdots, q_{n}\right)(x, t)$ are unknown functions of $x=\left(x_{1}, \cdots, x_{n}\right) \in \mathbb{R}^{n}(n \geq 1)$ and $t>0$. Typically, $u, q$ represent the velocity and radiating heat flux of the gas respectively.

The system (1.1) is a simplified version of the model for the motion of radiating gas in n-dimensional space. More precisely, in a certain physical situation, the system (1.1) gives a good approximation to the following system describing the motion of radiating gas, which is a quite general model for compressible gas dynamics where heat radiative transfer phenomena are taken into account,

$$
\left\{\begin{array}{l}
\rho_{t}+\operatorname{div}(\rho u)=0 \\
(\rho u)_{t}+\operatorname{div}(\rho u \otimes u+p I)=0 \\
\left\{\rho\left(e+\frac{|u|^{2}}{2}\right)\right\}_{t}+\operatorname{div}\left\{\rho u\left(e+\frac{|u|^{2}}{2}\right)+p u+q\right\}=0 \\
-\nabla \operatorname{div} q+a_{1} q+a_{2} \nabla \theta^{4}=0
\end{array}\right.
$$

where $\rho, u, p, e$ and $\theta$ are respectively the mass density, velocity, pressure, internal energy and absolute temperature of the gas, while $q$ is the radiative heat flux, and $a_{1}$ and $a_{2}$ are given positive constants depending on the gas itself. The first three equations are motivated by the usual Euler system, which describe the in-viscid flow of a compressible fluid and express conservation of mass, momentum and energy respectively. We refer to the book of Courant and Friedrichs [1] for a detailed derivation of several models in compressible gas-dynamics. The physical motivation of the fourth equation, which takes into account of heat radiation phenomena, is given in [2]. Moreover, the simplified model (1.1) was first recovered by Hamer (see [3]), and for the reduction of system (1.2) to system (1.1), see [2-4].

Concerning the investigation on the hyperbolic-elliptic coupled system in one-dimensional radiating gas, we refer to $[5,6]$. In the case of the muti-dimensional case, Francesco in [7] obtained the global well-posedness of the system (1.1) and analyzed the relaxation limits. Recently, in [8], Liu and Kawashima investigated the decay rate to diffusion wave for the initial value problem (1.1) in $n(n \geq 1)$-dimensional space by using a time-weighted energy method.

The rest of the paper is arranged as follows. Section 2 gives the full statement of our main theorem. In Section 3, we give estimates on the Green function by Fourier analysis which will be used in Section 5. Section 4 gives the global existence of solutions to the problem (2.3). In Section 5, we obtain the pointwise decay estimates of solutions.

Before closing this section, we give some notations to be used below. Let $\mathcal{F}[f]$ denote the Fourier transform of $f$ defined by 
$\mathcal{F}[f](\xi)=\hat{f}(\xi):=\frac{1}{(2 \pi)^{\frac{n}{2}}} \int_{\mathbb{R}^{n}} \mathrm{e}^{-i x \cdot \xi} f(x) \mathrm{d} x$, and we denote its inverse transform as $\mathcal{F}^{-1}$.

$L^{p}=L^{p}\left(\mathbb{R}^{n}\right)(1 \leq p \leq \infty)$ is the usual Lebesgue space with the norm \|\|$_{L^{p}}$. Let $S$ be a nonnegative integer, then $H^{s}=H^{s}\left(\mathbb{R}^{n}\right)$ denotes the Sobolev space of $L^{2}$ functions, equipped with the norm

$$
\|f\|_{H^{s}}:=\left(\sum_{k=0}^{s}\left\|\partial_{x}^{k} f\right\|_{L^{2}}^{2}\right)^{\frac{1}{2}} .
$$

For $k \geq 0$,

$\partial_{x}^{k}:=\left\{\partial_{x_{1}}^{k_{1}} \cdots \partial_{x_{n}}^{k_{n}} ; k_{i} \geq 0(1 \leq i \leq n), \sum_{i=1}^{n} k_{i}=k\right\}$. For nonnegative integer $k, C^{k}\left(I ; H^{s}\left(\mathbb{R}^{n}\right)\right)$ denotes the space of $k$-times continuously differentiable functions on the interval $I$ with values in the Sobolev space $H^{s}=H^{s}\left(\mathbb{R}^{n}\right)$.

Finally, in this paper, we denote every positive constant by the same symbol $C$ or $c$ without confusion. $[\cdot]$ is the Gauss's symbol.

\section{Main Theorems and Proof}

For simplicity, without loss of generality, we choose $a=\left(\frac{1}{2}, \cdots, \frac{1}{2}\right) \in \mathbb{R}^{n}$ in (1.1). That is, we will consider the following initial value problem:

$$
\begin{cases}u_{t}+\sum_{i=1}^{n} u u_{x_{i}}+\operatorname{div} q=0, & x \in \mathbb{R}^{n}, t>0, \\ -\nabla \operatorname{div} q+q+\nabla u=0, & x \in \mathbb{R}^{n}, t>0, \\ u(x, 0)=u_{0}(x), & x \in \mathbb{R}^{n} .\end{cases}
$$

Our Main results are the following:

Theorem 2.1. Let $s \geq\left\{\begin{array}{l}3, \quad n=1, \\ {[n / 2]+2, n \geq 2,}\end{array}\right.$ be an integer.

Assume that $u_{0} \in H^{s}\left(\mathbb{R}^{n}\right) \cap L^{1}\left(\mathbb{R}^{n}\right)$, and put $E_{0}:=\left\|u_{0}\right\|_{H^{s}}+\left\|u_{0}\right\|_{L^{1}}$. Then there is a small positive constant $\delta_{0}(<1)$ such that if $E_{0} \leq \delta_{0}$, then the problem (2.3) has a unique global solution $u(x, t)$ with

$$
\begin{gathered}
u \in C\left([0, \infty) ; H^{s}\left(\mathbb{R}^{n}\right)\right), \nabla u \in L^{2}\left([0, \infty) ; H^{s-1}\left(\mathbb{R}^{n}\right)\right), \\
q \in C\left([0, \infty) ; H^{s+1}\left(\mathbb{R}^{n}\right)\right) \cap L^{2}\left([0, \infty) ; H^{s+1}\left(\mathbb{R}^{n}\right)\right) .
\end{gathered}
$$

Moreover, if $s \geq[n / 2]+6$, and for any multi-indexes $\alpha$ with $|\alpha|<s-[n / 2]-1$, there exists some constant $r>\frac{n}{2}$ such that $\left|D_{x}^{\alpha} u_{0}(x)\right| \leq C E_{0}\left(1+|x|^{2}\right)^{-r}$, then for any $|\alpha| \leq s-[n / 2]-4$, the solution to Equation (2.3) has the following decay estimate,

$\left|D_{x}^{\alpha} u(x, t)\right| \leq C E_{0}(1+t)^{-\frac{n+|\alpha|}{2}}\left(1+\frac{|x|^{2}}{1+t}\right)^{-r}$. We also have the following corollary by using Theorem 2.1.

Corollary 2.2. Under the same assumptions in Theorem 2.1, the solution satisfies the following decay estimates:

$\left\|\partial_{x}^{k} u(t)\right\|_{L^{2}} \leq C E_{0}(1+t)^{-\frac{n}{4}-\frac{k}{2}}$, with $k$ satisfying

$0 \leq k \leq s-\left[\frac{n}{2}\right]-4$;

$\left\|\partial_{x}^{k} q(t)\right\|_{L^{2}} \leq C E_{0}(1+t)^{-\frac{n}{4}-\frac{k+1}{2}}$,

with $k$ satisfying $0 \leq k \leq s-\left[\frac{n}{2}\right]-5$.

Remark. In Theorem 2.1, we do not need to assume that $u_{0} \in L^{1}\left(\mathbb{R}^{n}\right)$, if $n \geq 2$. The results in Corollary 2.2 is similar to those in [7].

\section{The Global Existence of Solution}

This section is devoted to prove the global existence result stated in Theorem 2.1. In [7], the global existence of solutions to the problem (2.3) is obtained, but for the completeness of this paper, here we give the sketch of the proof.

Since a local existence result can be obtained by the standard method based on the successive approximation sequence, we omit its details and only derive the desired a priori estimates of solutions.

From $(2.3)_{1}-\Delta(2.3)_{1}-\operatorname{div}(2.3)_{2}$, we get that

$$
u_{t}-\Delta u_{t}-\Delta u+\sum_{i=1}^{n} u u_{x_{i}}-\Delta\left(\sum_{i=1}^{n} u u_{x_{i}}\right)=0 \text {. }
$$

Now we make energy estimates by using (3.4) under the following a priori estimate:

$$
\left\|\partial_{x} u(t)\right\|_{L^{\infty}} \leq \delta_{1}
$$

here $\delta_{1}<1$ is a given constant.

Multiplying (3.4) by $u$ and integrating with respect to $x$, by integration by parts we have that

$$
\frac{\mathrm{d}}{\mathrm{d} t}\left\{\|u(t)\|_{H^{1}}^{2}\right\}+\|\nabla u(t)\|_{L^{2}}^{2} \leq C\left\|\partial_{x} u(t)\right\|_{L^{\infty}}\|\nabla u(t)\|_{L^{2}}^{2} .
$$

Multiplying $\partial_{x}^{l}(3.4)$ by $\partial_{x}^{l} u(l \geq 1)$ and integrating with respect to $x$, by integration by parts we have that

$$
\begin{aligned}
& \frac{\mathrm{d}}{\mathrm{d} t}\left\{\left\|\partial_{x}^{l} u(t)\right\|_{H^{1}}^{2}\right\}+\left\|\partial_{x}^{l} \nabla u(t)\right\|_{L^{2}}^{2} \\
& \quad \leq C\left\|\partial_{x} u(t)\right\|_{L^{\infty}}\left\|\partial_{x}^{l} u(t)\right\|_{H^{1}}^{2}, l \geq 1 .
\end{aligned}
$$

We add up (3.7) with $1 \leq l \leq s-1$ and get that 


$$
\begin{aligned}
& \frac{\mathrm{d}}{\mathrm{d} t}\left\{\left\|\partial_{x} u(t)\right\|_{H^{s-1}}^{2}\right\}+\left\|\partial_{x}^{2} u(t)\right\|_{H^{s-2}}^{2} \\
& \quad \leq C\left\|\partial_{x} u(t)\right\|_{L^{\infty}}\left\|\partial_{x} u(t)\right\|_{H^{s-1}}^{2} .
\end{aligned}
$$

Combining (3.6) with (3.8) we have that

$$
\begin{aligned}
& \frac{\mathrm{d}}{\mathrm{d} t}\left\{\|u(t)\|_{H^{s}}^{2}\right\}+\left\|\partial_{x} u(t)\right\|_{H^{s-1}}^{2} \\
& \quad \leq C\left\|\partial_{x} u(t)\right\|_{L^{\infty}}\left\|\partial_{x} u(t)\right\|_{H^{s-1}}^{2} .
\end{aligned}
$$

In view of (3.5), (3.9) yields that

$$
\|u(t)\|_{H^{s}}^{2}+\int_{0}^{t}\left\|\partial_{x} u(\tau)\right\|_{H^{s-1}}^{2} \mathrm{~d} \tau \leq C\left\|u_{0}\right\|_{H^{s}}^{2} .
$$

From $(2.3)_{2}$ we have that $q=-(1-\Delta)^{-1} \nabla u$, thus (3.10) yields that

$$
\|q(t)\|_{H^{s+1}}^{2}+\int_{0}^{t}\left\|\partial_{x} q(\tau)\right\|_{H^{s+1}}^{2} \mathrm{~d} \tau \leq C\left\|u_{0}\right\|_{H^{s}}^{2}
$$

By the continuity argument, we have the following result.

Theorem 3.1. Let $s \geq\left[\frac{n}{2}\right]+2$ be an integer. Assume that $u_{0} \in H^{s}\left(\mathbb{R}^{n}\right)$, and put $E_{0}:=\left\|u_{0}\right\|_{H^{s}}$. Then there is a small positive constant $\delta_{0}(<1)$ such that if $E_{0} \leq \delta_{0}$, then the problem (2.3) has a unique global solution $u(x, t)$ with

$$
\begin{gathered}
u \in C\left([0, \infty) ; H^{s}\left(\mathbb{R}^{n}\right)\right), \nabla u \in L^{2}\left([0, \infty) ; H^{s-1}\left(\mathbb{R}^{n}\right)\right), \\
q \in C\left([0, \infty) ; H^{s+1}\left(\mathbb{R}^{n}\right)\right) \cap L^{2}\left([0, \infty) ; H^{s+1}\left(\mathbb{R}^{n}\right)\right) .
\end{gathered}
$$

\section{Estimates on Green Function}

In order to study the problem (2.3), we start with the Green function (or the fundamental solution) to the linear problem corresponding to the Equation (3.4), which satisfies

$$
\begin{cases}G_{t}-\Delta G_{t}-\Delta G=0, & x \in \mathbb{R}^{n}, t>0, \\ G(x, 0)=\delta(x), & x \in \mathbb{R}^{n}\end{cases}
$$

By Fourier transform we get that,

$$
\begin{cases}\hat{G}_{t}-\Delta \hat{G}_{t}-\Delta \hat{G}=0, & \xi \in \mathbb{R}^{n}, t>0 \\ \hat{G}(\xi, 0)=1, & \xi \in \mathbb{R}^{n}\end{cases}
$$

By direct calculation we have that $\hat{G}(\xi, t)=\mathrm{e}^{-\frac{|\xi|^{2} t}{1+|\xi|^{2}}}$. Let $\quad \chi_{1}(\xi)=\left\{\begin{array}{ll}1, & |\xi| \leq \varepsilon, \\ 0, & |\xi| \geq 2 \varepsilon,\end{array} \quad \chi_{3}(\xi)=\left\{\begin{array}{ll}1, & |\xi| \geq R, \\ 0, & |\xi| \leq R-1,\end{array}\right.\right.$ be the smooth cut-off functions, where $\varepsilon$ and $R$ are any fixed positive numbers satisfying $0<\varepsilon \ll 1 \ll R$. Set $\chi_{2}(\xi)=1-\chi_{1}(\xi)-\chi_{3}(\xi)$, and
$\hat{G}_{i}(\xi, t)=\chi_{i}(\xi) \hat{G}(\xi, t), \quad i=1,2,3$. We are going to study $G_{i}(x, t)$, which is the inverse Fourier transform of $\hat{G}_{i}(\xi, t)$.

First we give a lemma which is important for us to make estimates on the low frequency part.

Lemma 4.1. If $\hat{f}(\xi, t) \in C^{2 N}\left(\mathbb{R}^{n}\right)$ has compact support in the variables $\xi, N$ is a positive integer, and there exists a constant $b>0$, such that $\hat{f}(\xi, t)$ satisfies

$$
\begin{aligned}
& \left|D_{\xi}^{\beta}\left(\xi^{\alpha} \hat{f}(\xi, t)\right)\right| \\
& \leq C\left(|\xi|^{(|\alpha|+k-|\beta|)_{+}}+(1+t)^{\frac{|\beta|}{2}} t^{-\frac{|\alpha|+k}{2}}+|\xi|^{\alpha \mid+k}(1+t)^{\frac{|\beta|}{2}}\right) \\
& \times\left(1+t|\xi|^{2}\right)^{m} \mathrm{e}^{-b|\xi|^{2} t},
\end{aligned}
$$

for any multi-indexes $\alpha, \beta$ with $|\beta| \leq 2 N$, then

$$
\left|D_{x}^{\alpha} f(x, t)\right| \leq C_{N} t^{-\frac{n+|\alpha|+k}{2}} B_{N}(|x|, t),
$$

where $k$ and $m$ are any fixed integers,

$(a)_{+}=\max \{0, a\}$, and $B_{N}(|x|, t)=\left(1+\frac{|x|^{2}}{1+t}\right)^{-N}$.

Proof. If $|\beta| \leq k+|\alpha|$, by direct calculation we have that

$$
\begin{aligned}
& \left|x^{\beta} D^{\alpha} f(x, t)\right|=C\left|\int_{\mathbb{R}^{n}} \mathrm{e}^{\sqrt{-1} x \xi} D^{\beta} \xi^{\alpha} \hat{f}(\xi, t) \mathrm{d} \xi\right| \\
& \leq C \int_{\mathbb{R}^{n}}\left(|\xi|^{|\alpha|+k-|\beta|}+(1+t)^{\frac{|\beta|}{2}} t^{-\frac{\mid \alpha++k}{2}}+|\xi|^{|\alpha|+k}(1+t)^{\frac{|\beta|}{2}}\right) \\
& \times\left(1+t|\xi|^{2}\right)^{m} \mathrm{e}^{-b|\xi|^{2} t} \mathrm{~d} \xi \leq C(1+t)^{\frac{|\beta|}{2}} t^{-\frac{|\alpha|+n+k}{2}} .
\end{aligned}
$$

If $|\beta| \geq k+|\alpha|$, we also have

$$
\begin{aligned}
& \left|x^{\beta} D^{\alpha} f(x, t)\right|=C\left|\int_{\mathbb{R}^{n}} \mathrm{e}^{\sqrt{-1} x \xi} D^{\beta} \xi^{\alpha} \hat{f}(\xi, t) \mathrm{d} \xi\right| \\
& \leq C \int_{\mathbb{R}^{n}}\left(1+(1+t)^{\frac{|\beta|}{2}} t^{-\frac{|\alpha|+k}{2}}+|\xi|^{\alpha \mid+k}(1+t)^{\frac{|\beta|}{2}}\right)
\end{aligned}
$$

$$
\times\left(1+t|\xi|^{2}\right)^{m} \mathrm{e}^{-b|\xi|^{2} t} \mathrm{~d} \xi \leq C(1+t)^{\frac{|\beta|}{2} t^{-\frac{|\alpha|+n+k}{2}}} .
$$

Let $|\beta|=0$ when $|x|^{2} \leq 1+t$, and $|\beta|=2 N$ when $|x|^{2}>1+t$, we obtain from the above estimates that

$$
\left|D^{\alpha} f(x, t)\right| \leq C t^{-\frac{|\alpha|+n+k}{2}} \min \left\{1,\left(\frac{1+t}{|x|^{2}}\right)^{N}\right\} .
$$

Since $1+\frac{|x|^{2}}{1+t} \leq 2\left\{\begin{array}{ll}1, & |x|^{2} \leq 1+t, \\ \frac{|x|^{2}}{1+t}, & |x|^{2}>1+t,\end{array}\right.$, we have 


$$
\min \left\{1,\left(\frac{1+t}{|x|^{2}}\right)^{N}\right\} \leq \frac{2^{N}}{\left(1+\left(\frac{|x|^{2}}{1+t}\right)\right)^{N}}=2^{N} B_{N}(|x|, t) .
$$

Thus we complete the proof.

By using Lemma 4.1 we can get the following proposition about the estimates on $G_{1}(x, t)$.

Proposition 4.2. For sufficiently small $\varepsilon$, there exists constant $C>0$ such that

$$
\left|D_{x}^{\alpha} G_{1}(x, t)\right| \leq C_{N} t^{-\frac{n+|\alpha|}{2}} B_{N}(|x|, t) .
$$

Proof. For $|\xi|$ being sufficiently small, by noticing that $\hat{G}^{(\xi, t)}$ is a smooth function of $\xi$ near $|\xi|=0$ and using Taylor expansion we have that

$$
\begin{aligned}
\hat{G}^{(\xi, t)}= & \mathrm{e}^{-\frac{|\xi|^{2}}{1+|\xi|^{2}} t}=\mathrm{e}^{-|\xi|^{2} t+O\left(|\xi|^{4}\right) t} \text {. It yields that } \\
& \left|D_{\xi}^{\beta}(\xi \hat{G}(\xi, t))\right| \\
\leq & C\left(|\xi|^{(|\alpha|-|\beta|)_{+}}+(1+t)^{\frac{|\beta|}{2}} t^{-\frac{|\alpha|}{2}}+|\xi|^{|\alpha|}(1+t)^{\frac{|\beta|}{2}}\right) \\
& \times\left(1+t|\xi|^{2}\right)^{|\beta|} \mathrm{e}^{-\frac{|\xi|^{2} t}{2}} .
\end{aligned}
$$

Since

$$
\begin{aligned}
& \left|D_{\xi}^{\beta}\left(\xi^{\alpha} \widehat{G_{1}(\xi, t)}\right)\right| \\
& \quad=\sum_{\beta_{1}+\beta_{2}=\beta} \frac{\beta !}{\beta_{1} ! \beta_{2} !} D_{\xi}^{\beta_{1}} \chi_{1}(\xi) D_{\xi}^{\beta_{2}}\left(\xi^{\alpha} \hat{G}(\xi, t)\right),
\end{aligned}
$$

and $\left(|\alpha|-\left|\beta_{1}\right|\right)_{+} \geq(|\alpha|-|\beta|)_{+}$, we have that

$$
\begin{aligned}
& \left|D_{\xi}^{\beta}\left(\xi^{\alpha} \widehat{G}_{1}(\xi, t)\right)\right| \\
& \leq C\left(|\xi|^{\mid(\alpha|-| \beta \mid)_{+}}+(1+t)^{\frac{|\beta|}{2}} t^{-\frac{|\alpha|}{2}}+|\xi|^{|\alpha|}(1+t)^{\frac{|\beta|}{2}}\right) \\
& \quad \times\left(1+t|\xi|^{2}\right)^{|\beta|} \mathrm{e}^{-\frac{|\xi|^{2} t}{2}} .
\end{aligned}
$$

From Lemma 4.1 we obtain that

$$
\left|D_{x}^{\alpha} G_{1}(x, t)\right| \leq C_{N} t^{-\frac{n+|\alpha|}{2}} B_{N}(|x|, t) .
$$

Thus we complete the proof of Proposition 4.2.

As for $G_{2}(x, t)$ we have the following estimates.

Proposition 4.3. For fixed $\varepsilon$ and $R$, there exist positive numbers $m$ and $C$ such that

$$
\left|D_{x}^{\alpha} G_{2}(x, t)\right| \leq C \mathrm{e}^{-\frac{t}{2 m}} B_{N}(|x|, t) .
$$

Proof. For any fixed $\varepsilon$, we choose $m$ sufficiently large such that $m \geq 1+\frac{1}{\varepsilon^{2}}$. Since $\operatorname{supp} \widehat{G_{2}} \subseteq\{\xi ; \varepsilon \leq|\xi| \leq R\}$, we have that

$$
\left|\hat{G}_{2}(\xi, t)\right| \leq C \mathrm{e}^{-\frac{t}{m}} .
$$

It yields that $\left|D_{x}^{\alpha} G_{2}(x, t)\right| \leq C \mathrm{e}^{-\frac{t}{m}}$.

Now we shall give an estimate on $\left|x^{\beta} G_{2}(x, t)\right|$ by induction on $\beta$. Assume that, if $|\beta| \leq l-1$, then

$$
\left|D_{\xi}^{\beta} \hat{G}_{2}(\xi, t)\right| \leq C(1+t)^{|\beta|} \mathrm{e}^{-\frac{t}{m}},
$$

which is true as $|\beta|=0$ by (4.14)

By using (4.13), we have the following problem for $|\beta| \geq 1$,

$$
\left\{\begin{array}{l}
D_{\xi}^{\beta} \hat{G}(\xi, t)+\frac{|\xi|^{2}}{1+|\xi|^{2}} D_{\xi}^{\beta} \hat{G}(\xi, t)=-\left[D_{\xi}^{\beta}, \frac{|\xi|^{2}}{1+|\xi|^{2}}\right] \hat{G}(\xi, t), \\
\left.D_{\xi}^{\beta} \hat{G}(\xi, t)\right|_{t=0}=0 .
\end{array}\right.
$$

By multiplying (4.17), whose variables are now changed to $(\xi, s)$ by $\hat{G}(\xi, t-s)$ and integrating over the region $s \in(0, t)$, we have that

$$
D_{\xi}^{\beta} \hat{G}(\xi, t)=-\int_{0}^{t} \hat{G}(\xi, t-s)\left[D_{\xi}^{\beta}, \frac{|\xi|^{2}}{1+|\xi|^{2}}\right] \hat{G}(\xi, s) \mathrm{d} s .
$$

In view of (4.16) for $|\beta| \leq l-1$, it yields that

$$
\left|D_{\xi}^{\beta} \hat{G}(\xi, t)\right|_{\varepsilon|\xi| \xi R} \leq C \int_{0}^{t} \mathrm{e}^{-\frac{t-s}{m}}(1+t)^{|\beta|-1} \mathrm{e}^{-\frac{s}{m}} \mathrm{~d} s \leq C(1+t)^{|\beta|} \mathrm{e}^{-\frac{t}{m}},
$$

which shows that (4.16) is valid as $|\beta|=l$. This implies that, for $1 \leq|\beta| \leq l$,

$$
\begin{aligned}
& \left|x^{\beta} D_{x}^{\alpha} \partial_{t}^{h} G_{2}(x, t)\right| \\
& =C\left|\int_{\mathbb{R}^{n}} D_{\xi}^{\beta}\left(\xi^{\alpha} \chi_{2}(\xi) \hat{G}(\xi, t)\right) \mathrm{e}^{i x \cdot \xi} \mathrm{d} \xi\right| \\
& \leq C \mathrm{e}^{-\frac{t}{m}} \int_{\{\varepsilon \leq \xi \mid \leq R\}}(1+|\xi|)^{|\alpha|}(1+t)^{|\beta|} \mathrm{d} \xi \\
& \leq C(1+t)^{|\beta|} \mathrm{e}^{-\frac{t}{m}} .
\end{aligned}
$$

By using (4.15) when $|x|^{2} \leq 1+t$ and (4.18) with $|\beta|=2 N$ when $|x|^{2} \geq 1+t$, as well as the fact that $1+\frac{|x|^{2}}{1+t} \leq\left\{\begin{array}{ll}2, & |x|^{2} \leq 1+t, \\ \frac{2|x|^{2}}{1+t}, & |x|^{2}>1+t,\end{array}\right.$ we get that

$$
\left|D_{x}^{\alpha} G_{2}(x, t)\right| \leq C \mathrm{e}^{-\frac{t}{2 m}} B_{N}(|x|, t) .
$$


Thus we complete the proof of Proposition 4.3.

Next we will come to consider $G_{2}(x, t)$ First we give a lemmas which is useful in dealing with the high frequency part.

Lemma 4.4. If $\operatorname{supp} \hat{f}(\xi) \subset O_{R}:=\{\xi ;|\xi|>R\}$, and $\hat{f}(\xi)$ satisfies

$$
|\hat{f}(\xi)| \leq C,\left|D_{\xi}^{\beta} \hat{f}(\xi)\right| \leq C|\xi|^{-1-|\beta|},|\beta| \geq 1,
$$

then there exist distributions $f_{1}(x), f_{2}(x)$, and constant $C_{0}$ such that $f(x)=f_{1}(x)+f_{2}(x)+C_{0} \delta(x)$, where $\delta(x)$ is the Dirac function. Furthermore, for positive integer $2 N>n+|\alpha|,\left|D_{x}^{\alpha} f_{1}(x)\right| \leq C\left(1+|x|^{2}\right)^{-N}$,

$$
\left\|f_{2}\right\|_{L^{1}} \leq C, \operatorname{supp} f_{2}(x) \subset\left\{x ;|x|<2 \varepsilon_{0}\right\},
$$

with $\varepsilon_{0}$ being sufficiently small.

The proof of Lemma 4.4 can be seen in [9].

Choose sufficiently large $R$ such that

$\frac{|\xi|^{2}}{1+|\xi|^{2}}>\frac{1}{2}$, if $|\xi| \geq R-1$. By Taylor expansion, we have that

$$
\hat{G}_{3}(\xi, t)=\chi_{3}(\xi) \mathrm{e}^{-\left(1-\frac{1}{|\xi|^{2}}+O\left(\frac{1}{|\xi|^{4}}\right)\right) t} .
$$

It is obvious that $\left|\hat{G}_{3}(\xi, t)\right| \leq C \mathrm{e}^{-\frac{t}{2}}$. Since

$$
D_{\xi}^{\beta} \hat{G}_{3}(\xi, t)=C \sum_{\beta_{1}+\beta_{2}=\beta} D_{\xi}^{\beta_{1}} \chi_{3}(\xi) D_{\xi}^{\beta_{2}} \mathrm{e}^{-\left(1-\frac{1}{|\xi|^{2}}+O\left(\frac{1}{|\xi|^{4}}\right)\right) t},
$$

by direct calculation, we have that for $|\beta| \geq 1$,

$$
\left|D_{\xi}^{\beta} \hat{G}_{3}(\xi, t)\right| \leq C \mathrm{e}^{-\frac{t}{2}}|\xi|^{-|\beta|-1} .
$$

By using Lemma 4.4 we have the following result.

Proposition 4.5. For $R$ being sufficiently large, there exist distributions $G_{31}(x, t), G_{32}(x, t)$, and constant $C_{0}$ such that

$$
G_{3}(x, t)=\mathrm{e}^{-\frac{t}{2}}\left(G_{31}(x, t)+G_{32}(x, t)+C_{0} \delta(x)\right),
$$

where $\delta(x)$ is the Dirac function. Furthermore, for positive integer $2 N>n+|\alpha|$, the following estimates hold:

$$
\begin{gathered}
\left|D_{x}^{\alpha} G_{31}(x, t)\right| \leq C\left(1+|x|^{2}\right)^{-N}, \text { and } \\
\left\|G_{32}(., t)\right\|_{L^{1}} \leq C, \operatorname{supp} G_{32}(., t) \subseteq\left\{x ;|x| \leq 2 \varepsilon_{0}\right\},
\end{gathered}
$$

here $\varepsilon_{0}$ is sufficiently small.

Combining Proposition 4.2, Proposition 4.3, and Proposition 4.5 , we have the following theorem on the Green function.

Theorem 4.6. For any multi-indexes $\alpha$, there exists a distribution $\quad K(x, t)=\mathrm{e}^{-\frac{t}{2}}\left(G_{32}(x, t)+C_{0} \delta(x)\right), \quad$ such that the following estimate holds:

$$
\left|D_{x}^{\alpha}(G-K)(x, t)\right| \leq C(1+t)^{-\frac{n+|\alpha|}{2}} B_{N}(|x|, t),
$$

here, $N>\frac{n+|\alpha|}{2}$ is an arbitrary positive integer.

\section{Pointwise Estimates}

In this section, we focus on the pointwise estimates of solutions to the problem (2.3).

By Duhamel principle, the solution to the Equation (3.4) with initial datum $u(x, 0)=u_{0}(x)$ can be expressed as following,

$$
\begin{aligned}
u(x, t) & =G(., t) * u_{0}(x)-\int_{0}^{t} G(t-\tau) *(1-\Delta) \sum_{n=1}^{n} u u_{x_{i}}(\tau) \mathrm{d} \tau \\
& =: \bar{u}(x, t)+\tilde{u}(x, t) .
\end{aligned}
$$

Now we give a lemma which will be used in the following analysis.

Lemma 5.1. When $n_{1}, n_{2}>n / 2$, and $n_{3}=\min \left(n_{1}, n_{2}\right)$, we have that

$$
\int_{\mathbb{R}^{n}}\left(1+\frac{|x-y|^{2}}{1+t}\right)^{-n_{1}}\left(1+|y|^{2}\right)^{-n_{2}} \mathrm{~d} y \leq C\left(1+\frac{|x|^{2}}{1+t}\right)^{-n_{3}} .
$$

The proof of Lemma 5.1 can be seen in [9].

Since $\bar{u}(x, t)=(G-K)(x, t)+K(x, t)=: I_{1}+I_{2}$, by using Lemma 5.1 and Theorem 4.6 with $N>r$, we have that

$$
\left|D_{x}^{\alpha} I_{1}\right| \leq C E_{0}(1+t)^{-\frac{n+|\alpha|}{2}} B_{r}(|x|, t) .
$$

Noticing that if $|x-y| \leq 2 \varepsilon_{0}$, then $\left(1+|y|^{2}\right)^{-1} \leq C\left(1+|x|^{2}\right)^{-1}$, we have that

$$
\begin{aligned}
\left|D_{x}^{\alpha} I_{2}\right| & =\mathrm{e}^{-\frac{t}{2}}\left|\int_{\mathbb{R}^{n}}\left(G_{32}(x-y, t)+C_{0} \delta(x-y)\right) D_{x}^{\alpha} u_{0}(y) \mathrm{d} y\right| \\
& \leq C E_{0} \mathrm{e}^{-\frac{t}{2}} B_{r}(|x|, t) .
\end{aligned}
$$

Thus we obtain that

$$
\begin{aligned}
& \left|D_{x}^{\alpha} \bar{u}(x, t)\right| \leq C E_{0}(1+t)^{-\frac{n+|\alpha|}{2}} \\
& \cdot B_{r}(|x|, t),|\alpha|<s-\left[\frac{n}{2}\right]-1 .
\end{aligned}
$$

Next we come to make estimates on $\tilde{u}(x, t)$. To this end, we will use the following lemma.

Lemma 5.2. Assume $n \geq 1$, then the following inequalities hold,

1) If $\tau \in[0, t]$, and $A^{2} \geq t$, then 


$$
\left(1+\frac{A^{2}}{1+\tau}\right)^{-n} \leq 2^{n}\left(\frac{1+\tau}{1+t}\right)^{n}\left(1+\frac{A^{2}}{1+t}\right)^{-n}
$$

2) If $A^{2} \leq t$, then $1 \leq 2^{n}\left(1+\frac{A^{2}}{1+t}\right)^{-n}$.

$$
\varphi_{\alpha}(x, t):=(1+t)^{\frac{n+|\alpha|}{2}}\left(B_{r}(|x|, t)\right)^{-1},
$$

Denote $M(T):=\sup _{\substack{(x, \tau) \in \mathbb{R}^{n} \times[0, T) \\[n]}}\left\{\left|D_{x}^{\alpha} u(x, \tau)\right| \varphi_{\alpha}(x, \tau)\right\}$. $|\alpha|<s-\left[\frac{n}{2}\right]-4$

Now we come to make estimates to $\tilde{u}(x, t)$ by using

Theorem 4.6 with $N>r$ and Lemma 5.2. We decompose $D_{x}^{\alpha} \tilde{u}(x, t)$ as following,

$$
\begin{aligned}
& D_{x}^{\alpha} \tilde{u}(x, t) \\
& =\int_{0}^{\frac{t}{2}} \int_{\{y ;|x| \geq 2|y|\}} \sum_{i=1}^{n} \partial_{x_{i}} D_{x}^{\alpha}(1-\Delta)(G-K)(x-y, t-\tau) u^{2}(y, \tau) \mathrm{d} y \mathrm{~d} \tau \\
& +\int_{0}^{\frac{t}{2}} \int_{\{y ;|x| \leq 2|y| y} \sum_{i=1}^{n} \partial_{x_{i}} D_{x}^{\alpha}(1-\Delta)(G-K)(x-y, t-\tau) u^{2}(y, \tau) \mathrm{d} y \mathrm{~d} \tau \\
& +\frac{1}{2} \int_{\frac{t}{2}}^{t} \int_{\{y ;|x| \geq 2|y|\}} \sum_{i=1}^{n} \partial_{x_{i}}(1-\Delta)(G-K)(x-y, t-\tau) D_{y}^{\alpha}\left(u^{2}\right)(y, \tau) \mathrm{d} y \mathrm{~d} \tau \\
& +\frac{1}{2} \int_{\frac{t}{2}}^{t} \int_{\{y ;|x| \leq 2|y|\}} \sum_{i=1}^{n} \partial_{x_{i}}(1-\Delta)(G-K)(x-y, t-\tau) D_{y}^{\alpha}\left(u^{2}\right)(y, \tau) \mathrm{d} y \mathrm{~d} \tau \\
& +\int_{0}^{t} \int_{R^{n}} K(x-y, t-\tau) D_{y}^{\alpha}(1-\Delta) \sum_{i=1}^{n}\left(u u_{x_{i}}\right)(y, \tau) \mathrm{d} y \mathrm{~d} \tau \\
& =: I_{31}+I_{32}+I_{41}+I_{42}+I_{5} .
\end{aligned}
$$

Next we estimate $I_{i}(i=1,2,3,4,5)$ respectively by using Theorem 4.6. By using Lemma 5.2 (1), we have that

$$
\begin{aligned}
\left|I_{31}\right| & \leq C M(T)^{2} \int_{0}^{\frac{t}{2}} \int_{\{y ;|x| \geq 2|y|\}}(1+t-\tau)^{-\frac{n+|\alpha|+1}{2}} B_{N}(|x-y|, t-\tau) \times(1+\tau)^{-n} B_{r}(|y|, \tau) \mathrm{d} y \mathrm{~d} \tau \\
& \leq C M(T)^{2}(1+t)^{-\frac{n+|\alpha|+1}{2}} B_{N}(|x|, t) \times \int_{0}^{\frac{t}{2}} \int_{\{y|x| x|2| y \mid\}}(1+\tau)^{-n} B_{r}(|y|, \tau) \mathrm{d} y \mathrm{~d} \tau \\
& \leq C M^{2}(T) B_{r}(|x|, t)(1+t)^{-\frac{n+|\alpha|}{2}}
\end{aligned}
$$

Now we estimate $I_{32}$. in two cases.

Case 1. $|x|^{2} \geq t$. By using Lemma 5.2 (1), we have that

$$
\begin{aligned}
\left|I_{32}\right| & \leq C M(T)^{2} \int_{0}^{\frac{t}{2}} \int_{\{y ;|x| \leq 2|y|\}}(1+t-\tau)^{-\frac{n+|\alpha|+1}{2}} B_{N}(|x-y|, t-\tau) \times(1+\tau)^{-n} B_{r}(|y|, \tau) \mathrm{d} y \mathrm{~d} \tau \\
& \leq C M(T)^{2}(1+t)^{-\frac{n+|\alpha|+1}{2}} \int_{0}^{\frac{t}{2}} \int_{\{y|x| x|\leq 2| y \mid\}} B_{N}(|x-y|, t) \times(1+\tau)^{-n} B_{r}(|x|, t)\left(\frac{1+\tau}{1+t}\right)^{\frac{n}{2}} \mathrm{~d} y \mathrm{~d} \tau \\
& \leq C M(T)^{2} B_{r}(|x|, t)(1+t)^{-\frac{n+|\alpha|}{2}} .
\end{aligned}
$$

Case 2. $|x|^{2}<t$. By using Lemma 5.2 (2), we have that

$$
\begin{aligned}
& \left|I_{32}\right| \leq C M(T)^{2} \int_{0}^{\frac{t}{2}} \int_{\{y|x| x|\leq 2| y \mid\}}(1+t-\tau)^{-\frac{n+|\alpha|+1}{2}} \\
& \times B_{N}(|x-y|, t-\tau) \times(1+\tau)^{-n} B_{r}(|y|, \tau) \mathrm{d} y \mathrm{~d} \tau \\
& \leq C M(T)^{2}(1+t)^{-\frac{n+|\alpha|+1}{2}} \int_{0}^{\frac{t}{2}}(1+\tau)^{-n} \\
& \times B_{r}(|y|, \tau) \mathrm{d} \tau \leq C M(T)^{2} B_{r}(|x|, t)(1+t)^{-\frac{n+|\alpha|}{2}} .
\end{aligned}
$$

Combining the two cases, we have that 


$$
\left|I_{32}\right| \leq C M(T)^{2} B_{r}(|x|, t)(1+t)^{-\frac{n+|\alpha|}{2}} .
$$

As for $I_{41}$, we also need to divide it into two cases.

Case 1. $|x|^{2} \geq t$. By using Lemma 5.2 (1), we have that

$$
\begin{aligned}
\left|I_{41}\right| \leq & C M(T)^{2} \int_{\frac{t}{2}}^{t} \int_{\{y ; x|x| \geq 2|y|\}}(1+t-\tau)^{-\frac{n+1}{2}} B_{N}(|x-y|, t-\tau) \\
& \times(1+\tau)^{-\frac{2 n+|\alpha|}{2}} B_{r}(|y|, \tau) \mathrm{d} y \mathrm{~d} \tau \\
\leq & C M(T)^{2}(1+t)^{-\frac{2 n+|\alpha|}{2}} \int_{\frac{t}{2}}^{t} \int_{\{y ;|x| \geq 2|y|\}}(1+t-\tau)^{-\frac{n+1}{2}} \\
& \times B_{N}(|x|, t)\left(\frac{1+t-\tau}{1+t}\right)^{\frac{n}{2}} B_{r}(|y|, t) \mathrm{d} y \mathrm{~d} \tau \\
\leq & C M(T)^{2} B_{r}(|x|, t)(1+t)^{-\frac{n+|\alpha|}{2}} .
\end{aligned}
$$

Case 2. $|x|^{2}<t$. By using Lemma 5.2 (2), we have that

$$
\begin{aligned}
& \left|I_{41}\right| \leq C M(T)^{2} \int_{\frac{t}{2}}^{t} \int_{\{y ;|x| z|y| y\}}(1+t-\tau)^{-\frac{n+1}{2}} B_{N}(|x-y|, t-\tau) \\
& \quad \times(1+\tau)^{-\frac{2 n+|\alpha|}{2}} B_{r}(|y|, \tau) \mathrm{d} y \mathrm{~d} \tau \\
& \leq C M(T)^{2}(1+t)^{-\frac{2 n+|\alpha|}{2}} \int_{\frac{t}{2}}^{t} \int_{\{y|x| x|2| y \mid\}}(1+t-\tau)^{-\frac{n+1}{2}} \\
& \quad \times B_{N}(|x-y|, t-\tau) \mathrm{d} y \mathrm{~d} \tau \\
& \leq C M(T)^{2} B_{r}(|x|, t)(1+t)^{-\frac{n+|\alpha|}{2}} .
\end{aligned}
$$

Combining the two cases, we have that

$$
\left|I_{41}\right| \leq C M(T)^{2} B_{r}(|x|, t)(1+t)^{-\frac{n+|\alpha|}{2}} .
$$

As for $I_{42}$, by direct calculation, we have that

$$
\begin{aligned}
\left|I_{42}\right| \leq & C M(T)^{2} \int_{\frac{t}{2}}^{t} \int_{\{y|x| x|\leq 2| y \mid\}}(1+t-\tau)^{-\frac{n+1}{2}} B_{N}(|x-y|, t-\tau) \\
& \times(1+\tau)^{-\frac{2 n+|\alpha|}{2}} B_{r}(|y|, \tau) \mathrm{d} y \mathrm{~d} \tau \\
\leq & C M(T)^{2}(1+\tau)^{-\frac{2 n+|\alpha|}{2}} B_{r}(|x|, t) \\
& \times \int_{\frac{t}{2}}^{t} \int_{\{y ;|x| \leq 2|y|\}}(1+t-\tau)^{-\frac{n+1}{2}} B_{N}(|x-y|, t-\tau) \mathrm{d} y \mathrm{~d} \tau \\
\leq & C M(T)^{2} B_{r}(|x|, t)(1+t)^{-\frac{n+|\alpha|}{2}} .
\end{aligned}
$$

To estimate $I_{5}$, we will use the following result, which is obtained in [7].

Lemma 5.3. 1) If $s \geq\left\{\begin{array}{ll}3, & n=1, \\ {\left[\frac{n}{2}\right]+2,} & n \geq 2,\end{array}\right.$ and $u_{0} \in H^{s}\left(\mathbb{R}^{n}\right) \cap L^{1}\left(\mathbb{R}^{n}\right)$, put $E_{0}:=\left\|u_{0}\right\|_{H^{s}}+\left\|u_{0}\right\|_{L^{1}}$, then the following estimate holds:

$$
\left\|\partial_{x}^{k} u(t)\right\|_{H^{s-k}} \leq C E_{0}(1+t)^{-\frac{n}{4}-\frac{k}{2}} \text {, with } 0 \leq k \leq s-1 .
$$

2) If $s \geq\left[\frac{n}{2}\right]+2$, and $u_{0} \in H^{s}\left(\mathbb{R}^{n}\right), n \geq 2$, put $E_{0}:=\left\|u_{0}\right\|_{H^{s}}$, then the following estimate holds: $\left\|\partial_{x}^{k} u(t)\right\|_{H^{s-k}} \leq C E_{0}(1+t)^{-\frac{k}{2}}$, with $0 \leq k \leq s$.

We estimate $I_{5}$ as following,

$$
\begin{aligned}
\left|I_{5}\right| \leq & C \int_{0}^{t} \mathrm{e}^{-\frac{t-\tau}{2}}\left|G_{32}(t-\tau) * D_{x}^{\alpha}(1-\Delta) \sum_{i=1}^{n}\left(u u_{x_{i}}\right)(\tau)\right| \mathrm{d} \tau \\
& +C \int_{0}^{t} \mathrm{e}^{-\frac{t-\tau}{2}}\left|D_{x}^{\alpha}(1-\Delta) \sum_{i=1}^{n}\left(u u_{x_{i}}\right)(x, \tau)\right| \mathrm{d} \tau .
\end{aligned}
$$

Notice that $D_{x}^{\alpha} \Delta \sum_{i=1}^{n} \partial_{x_{i}}\left(u^{2}\right)$ consists of terms of $\partial_{x}^{k_{1}} u \partial_{x}^{k_{2}} u, k_{1}, k_{2} \geq 0, k_{1}+k_{2}=|\alpha|+3$. Without loss of generality, we assume that $k_{1} \leq k_{2}$, then $k_{1} \leq \max \{|\alpha|, 2\}$. Since $s \geq\left[\frac{n}{2}\right]+6$ and $|\alpha| \leq s-\left[\frac{n}{2}\right]-4$, we have that

$$
\left|\partial_{x}^{k_{1}} u(y, \tau)\right| \leq M(T)(1+\tau)^{-\frac{n+k_{1}}{2}} B(|y|, \tau) .
$$

By using Gagliardo-Nirenberg inequality and Lemma 5.3 , we have that

$$
\left\|\partial_{x}^{k_{2}} u(\tau)\right\|_{L^{\infty}} \leq C E_{0}(1+\tau)^{-\frac{k_{2}}{2}}, k_{2} \leq s-\left[\frac{n}{2}\right]-1 .
$$

Thus we have that

$$
\left|\partial_{x}^{k_{1}} u(y, \tau) \partial_{x}^{k_{2}} u(y, \tau)\right| \leq C E_{0} M(T)(1+\tau)^{-\frac{n+k_{1}+k_{2}}{2}} \text {. }
$$

Combined with Proposition 4.5 and the fact that $\left(1+\frac{|y|^{2}}{1+t}\right)^{-1} \leq C\left(1+\frac{|x|^{2}}{1+t}\right)^{-1}$, if $|x-y| \leq 2 \varepsilon_{1}$, it yields that

$$
\begin{aligned}
\left|I_{5}\right| & \leq C E_{0} M(T) \int_{0}^{t} \mathrm{e}^{-\frac{t-\tau}{2}}(1+\tau)^{-\frac{n+|\alpha|+1}{2}} \mathrm{~d} \tau \\
& \leq C E_{0} M(T) B_{r}(|x|, t)(1+t)^{-\frac{n+|\alpha|}{2}} .
\end{aligned}
$$

Combining $I_{31}, I_{32}, I_{41}, I_{42}$ and $I_{5}$, we have that

$\left|D_{x}^{\alpha} \tilde{u}(x, t)\right| \leq C\left(E_{0} M(T)+M(T)^{2}\right)\left(\varphi_{\alpha, h}(x, t)\right)^{-1}$.

Proof of Theorem 2.1. In view of (5.19) and (5.20), we get that

$\left|D_{x}^{\alpha} u(x, t)\right| \leq C\left(E_{0}+E_{0} M(T)+M(T)^{2}\right)\left(\varphi_{\alpha, h}(x, t)\right)^{-1}$.

It yields that 


$$
M(T) \leq C\left(E_{0}+E_{0} M(T)+M(T)^{2}\right) .
$$

Thus if $E_{0}$ is suitably small, we obtain $M(T) \leq C E_{0}$ by the continuous dependence on the initial data. In view of Theorem 3.1, the proof of Theorem 2.1 is completed.

\section{Acknowledgements}

The first author is partially supported by the National Natural Science Foundation of China (Grant No. 11201142) and by the Fundamental Research Funds for the Central Universities (Grant No. 11QL40). The second author is partially supported by the National Natural Science Foundation of China (Grant No. 11201144).

\section{REFERENCES}

[1] R. Courant and K. O. Friedrichs, "Supersonic Flow and ShockWaves," Interscience Publishers, Inc., New York, 1948.

[2] W. G. Vincenti and C. H. Kruger, "Introduction to Physical Gas Dynamics," Wiley, New York, 1965.

[3] K. Hamer, "Nonlinear Effects on the Propogation of Sound Waves in a Radiating Gas," Quarterly Journal of Mechanics \& Applied Mathematics, Vol. 24, No. 2, 1971, pp. 155-168. http://dx.doi.org/10.1093/qjmam/24.2.155

[4] W. L. Gao and C. J. Zhu, "Asymptotic Decay toward the Planar Rarefaction Waves for a Model System of the Ra- diating Gas in Two Dimensions," Mathematical Models and Methods in Applied Sciences, Vol. 18, No. 4, 2008, pp. 511-541. http://dx.doi.org/10.1142/S0218202508002760

[5] S. Kawashima and S. Nishibata, "Weak Solutions with a Shock to a Model System of the Radiating Gas," Science Bulletin of Josai University, Vol. 5, Special Issue, 1998, pp. 119-130.

[6] S. Kawashima and S. Nishibata, "Cauchy Problem for a Model System of the Radiating Gas: Weak Solution with a Jump and Classical Solutions," Mathematical Models and Methods in Applied Sciences, Vol. 9, No. 1, 1999, pp. 69-91. http://dx.doi.org/10.1142/S0218202599000063

[7] M. D. Francesco, "Initial Value Problem and Relaxation Limits of the Hamer Model for Radiating Gases in Several Space Variables," Nonlinear Differential Equations and Applications NoDEA, Vol. 13, No. 5-6, 2007, pp. 531-562. http://dx.doi.org/10.1007/s00030-006-4023-y

[8] Y. Liu and S. Kawashima, "Asymptotic Behavior of Solutions to a Model System of a Radiating Gas," Communications on Pure and Applied Analysis, Vol. 10, No. 1, 2011, pp. 209-223.

http://dx.doi.org/10.3934/cpaa.2011.10.209

[9] W.-K. Wang and T. Yang, "The Pointwise Estimates of Solutions for Euler Equations with Damping in MultiDimensions," Journal of Differential Equations, Vol. 173, No. 2, 2001, pp. 410-450. http://dx.doi.org/10.1006/jdeq.2000.3937 